\title{
An Innovative Wearable Electronic Tourist Guide Device and Automatic Guide Service
}

\author{
Dawei Cai \\ Iwate Prefectural University \\ Iwate, Japan 020-0193
}

\begin{abstract}
This paper presents the design and implementation issues of an earphone style wearable device that may provide an automatic guidance service for tourists, and a synthesis management system that provides main guide device management functions, such as device recharging, guide contents installing, and tourists usage history collection. To provide tourists an automatic guidance service, it is necessary to identify the location and orientation of the tourist in real time. To identify the location based on signal from a NFC beacon tag, an approach that can eliminate the influences from adjacent tag and give correct location identification. To identify the orientation, a MEMS sensor that provides acceleration and terrestrial magnetism information is used, and an algorithm for orientation identification is proposed. If a tourist wants to know explanation about an exhibit, what he has to do is only move to the exhibit object and stands for a moment. Then the device will automatically recognize the status of the tourist, and start an explanation about the exhibit. Based the design, the prototype of the device has been developed on an ARM SoC and RTOS platform environment. The paper presents the total route of system design such as the main identification algorithms, and highlights the implementation approach.
\end{abstract}

\section{General Terms}

System Development

\section{Keywords}

Wearable Device, MEMS Sensor, Tourist application, Electronic tourist guide, Communication Protocol

\section{INTRODUCTION}

There is an increasing trend of interest in cultural tourist products in the world, and the World Tourism Organization expects that the cultural tourism market would be among the five most important segments of the tourist market in the future. There is no doubt that cultural tourism becomes more and more important form of tourism. Today's most popular tourist destinations are the ones which adjust their offer to tourist needs providing them a range of different activities and experiences. In the case, a cultural product would be a unique emotion, experience, for only sightseeing itself is not satisfactory [1]. Managers of the world known museums agreed that museums in the future have to make a balance between managing collections and visitors. Involving visitors into dialogue and using museums' collections creatively are now the most important tasks for museums' employees. In the future only museums that really connect with their audiences will be sustainable. Therefore, tourists should be invited to actively participate. One of the ways to realize such a thing is to accept and include some new technologies in museums exhibitions. They could be an important part of interaction between tourists and museum management side. In these years, more and more museums have tried mobile experiences and are considering to create mobile experiences in order to provide tourists more fulfilling and satisfied experiences.

In recent years, along with the spread of mobile phones, exhibition guide services using mobile phones are increasing [2], [3]. These services made it possible for individual commentary that tourists cannot provide with traditional paper style guidance. With the smartphone, multimedia contents can be used easily to explain the exhibition in detail or Multilanguage. This kind of service may be a great help for foreign tourists who do not know the local language. However, with such guidance using mobile phones, tourists need to operate the guide device with both hands. For example, you have to touch the screen or slide it. With their hands, they cannot do other work and become a heavy burden. Means are needed to open tourists' hands and provide tourist commentary automatically. Now, wearable device have attracted attention rapidly. Wearable device of spectacle type, wristwatch type and head type were developed. The merit of the wearable device is that even if this device is installed, the person freely performs other work. Therefore, if a tourist can wear a device such as a wearable device and use the tour guide without operation, the burden on the tourist can be greatly reduced. Near Filed Communication (NFC) is a communication technology that can provide a short range communication with small power consumption. We expect to use this communication to identify the location of the tourists. MEMS (Micro Electro Mechanical Systems) can provide various sensing information, such as acceleration and terrestrial magnetism. It is very small in size and can be implemented in a small PCB area. With information from NFC and MEMS, we can identify the position information in room, as this case, the GPS is not available. In order to provide a tourist a correct multimedia guidance related on his standing position and orientation, we have developed a multimedia portable device for interactive guidance [4], [5], [6], [7]. In the tourist facilities such as Japanese' museums, this device and management system have already been accepted for service 
in several exhibition faculties in Japan, and obtained a favorable comment from users.

Wireless charging technology is also attracting attention for mobile device and wearable devices. By using wireless charging, the burden of device management can be greatly reduced. Currently, wireless charging for mobile phones has already been put to practical use. As representative technology, there are inductive technology such as WPC (wireless power consortium), and electromagnetic field resonance technology. Using wireless charging makes it unnecessary to connect cables, making it very easy to manage devices.

In this paper, we developed a new earphone type wearable device and propose an approach to identify orientation and position of wearable devices by combining the position information from NFC and the orientation information from a 6 axis acceleration and terrestrial magnetism sensor, based on the previous work [?]. With the orientation and position information, the device may recognize the visitors' position and their orientation, and give an automatic guidance service for the exhibition object just in front of the exhibition objects. There is a wireless charging added to the device to take advantage of the convenience of wireless charging.

\section{AUTOMATIC GUIDANCE SYSTEM}

\subsection{System Configuration}

Fig 11 illustrates the system configuration of the earphone style wearable guide system. As shown in Fig 1 there are two main area, one is facilities management department, another is exhibition facilities. In the facilities management department, content editor provides content creation and editing and coding, and tourist usage history analysis application aggregates the behavior of tourists, and integrated device management station is used for setting content codes to the device and charging the device and read out the history data from the device. In exhibition facilities, several PAN tags or IR tags are located in exhibition area to indicate the location of the exhibit. The wearable devices are used to automatically play the guidance contents of the exhibition for tourists, when the wearable device approaches an exhibition. For the wearable is equipped on the visitor's head, the Mutual position between the device and user is fixed or constrained. Therefore, the orientation of the device can be used to show the orientation of the visitor.

For the earphone style wearable device is not at a regular and consists of curved surfaces in total. Therefore, it is not easy to install a connector for charging. To solve the problem, a wireless charging solution is applied in the integrated device management station. When the wearable device is inserted the slot in the station, the charging process will begin, while the tourist usage history information will be sent to the station with a hybrid communication which is a combination of $2.4 \mathrm{GHz} \mathrm{NFC}$ and $16 \mathrm{Mbps}$ infrared communication. We developed a very efficient approach for large capacity data transmitting to multiple devices at the same time, and the audio guide contents are transmitted to all the wearable devices which are inserted in the station.

\subsection{Earphone Style Wearable Guidance Device}

As shown in Fig 2 the wearable guidance device is designed as an earphone style that can make the two hands of tourist free. In the device, here are only three buttons for operation, two for volume adjustment and one for start or stop. A 6 axis acceleration and terrestrial magnetism sensor is boarded to get the orientation and attitude condition of the device. There is a NFC module to provide a wireless communication with $2.4 \mathrm{MHz}$ band. With the NFC, the

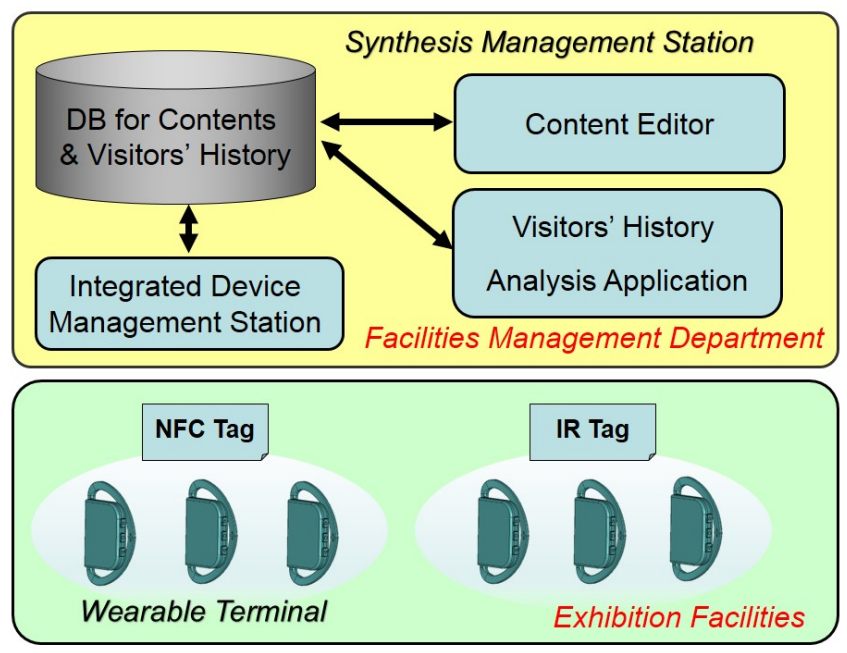

Fig. 1. The basic architecture of the guide system

device can receive signal from NFC beacon tag, and send data between the device and the management station. With the location identification algorithm and orientation algorithm, the wearable device can identify the status of the tourists, and automatically start playing the related content automatically without any operation by the tourists. Therefore the device can be used by a wide range visitor from children to elder persons, for it is no necessary to master the usage of the device before using it. The only thing that the visitors should to do is putting the device on their ear as shown in $\mathrm{FIg} 2$

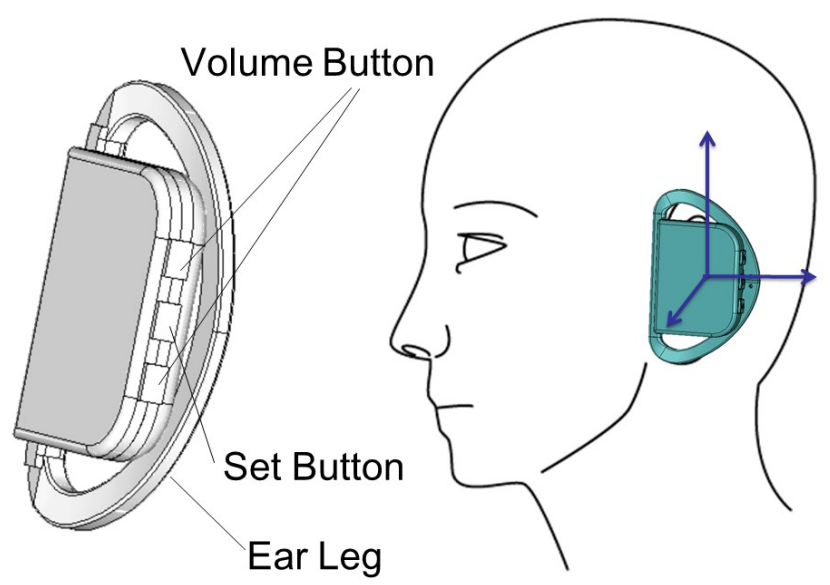

Fig. 2. The design of an earphone style wearable guide device

The new earphone style wearable guide device is developed with an ARM926 core SoC and a RTOS platform called ThreadX. Fig 3 shows the main structure of the wearable guide device. As shown in Fig 3 the wearable device is composed with a ARM926 core SoC, 6 degrees sensor IC, ubiquitous communication IC, a flash memory, a mini speaker and a wireless charging module. A $200 \mathrm{mAh} \mathrm{Li-iron}$ battery is used to provide the power to guarantee the device can 


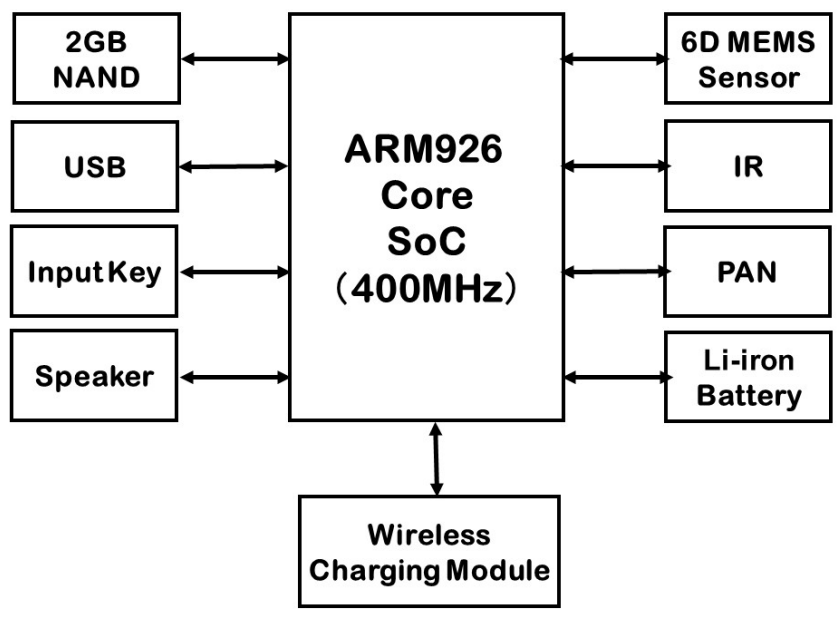

Fig. 3. The architecture of the wearable guide device

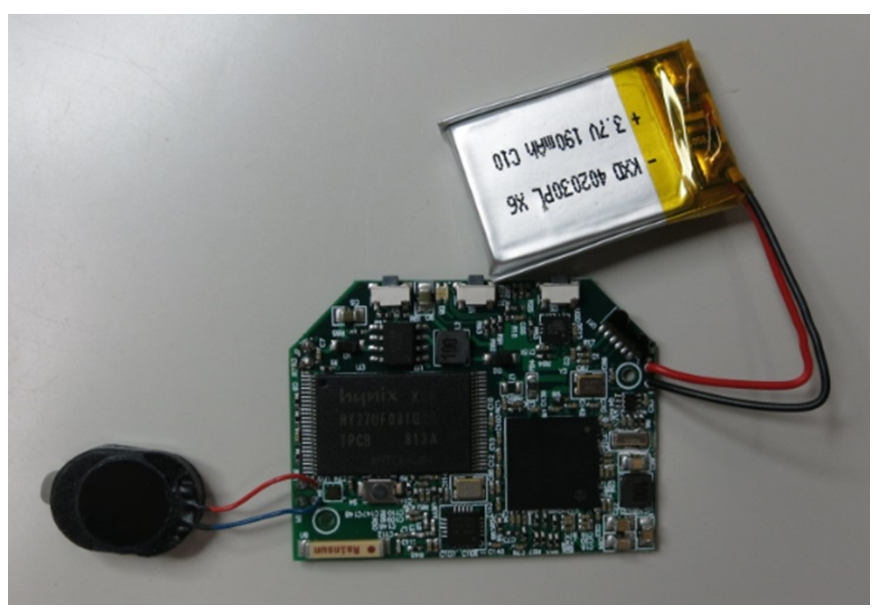

Fig. 4. The PCB module of the wearable guide device

work more than 8 hours without charging. Fig 4 shows the PCB module of the wearable guide device. There is a coil fixed on the PCB to provide a wireless charging function. Fig 5 shows a photo of the trial manufacture device. The weight of the device is less than $40 \mathrm{~g}$, it is light enough for wearing on the ear for a long time. As wireless communication, we developed our own communication protocol, which can reduce power consumption greatly compared with fashionable wireless communication such as $\mathrm{Wi}-\mathrm{Fi}$, Bluetooth. Therefore, the new wearable guide can provide a much longer operation time. For a guide system in exhibition facilities, it is important that the wearable guide device can provide guidance service without charging for the total opening period of tourist facilities. In the design of the wearable device, it has become important to extremely reduce the power consumption of the communication function and the reproduction function in order to realize long-time operation and weight saving.

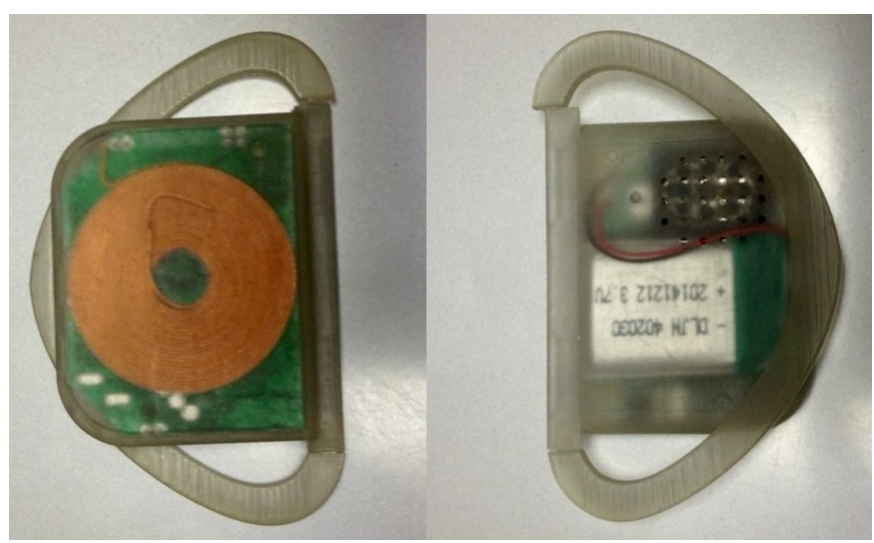

Fig. 5. The trial manufacture of the earphone style wearable guide device

\subsection{Wireless Charging}

As shown in Fig 2 the shape of the earphone style wearable guide device is not easy to provide a direct connection for charging, so we adopt wireless charging technology in the device. In our wireless charging circuit, we adopted inductive technology for its high efficiency and low cost. To realize the wireless function, we designed a charger slot to provide wireless charging as shown in Fig 6 The shape of the charging slot is designed according to the outer shape of the wearable device, and the charging distance of the charging coil can be reliably secured. With such a design, the management load of charging is greatly reduced, contributing to cost reduction of the operation side. When you want to charge the wearable device for low battery condition, the only thing you should do is putting the wearable device into the slot of the charger. The wireless charger can provide up to $250 \mathrm{~mA}$ charging current, and charging time is less than three hours to charge the battery to full.

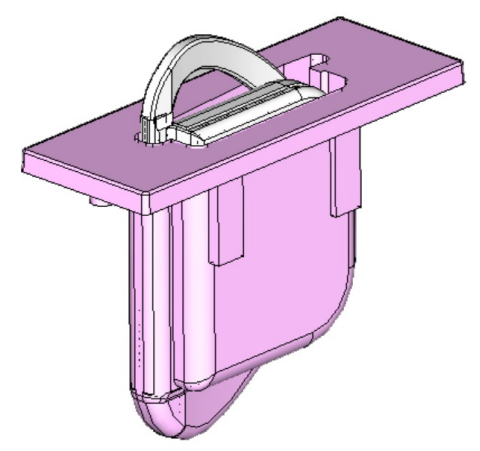

Fig. 6. The design of the wireless charger and the device 


\section{IDENTIFICATION OF POSITION AND STATUS}

In order to provide tourists with correct guidance at the right time, it is important to accurately identify the location, orientation and movement of tourists in real time.

Here, regarding the position information, a packet transmitted from the NFC tag installed in the exhibit or its vicinity is used. In the method of obtaining position information using a tag such as a beacon, a method using the reception strength of radio waves becomes mainstream. However, in reality, the radio field strength is enlarged to the movement of the surroundings, the change of the exhibition arrangement, the change of the weather, etc., and seems to be problematic in practical terms.

\subsection{Identification Approach of Position}

When there are a multiple exhibition spot in the exhibition space, it is necessary to use multiple beacon tags to indicate each exhibition spot area. For the wave signal from the tag may reach the other area, a wearable device may receive the signals from the tags that are in other spot area. In this case, the identification of the spot will be an error result.

In the location-specific application using the wireless beacon, there is a method of measuring the signal strength of the wireless tag. Since this signal intensity varies greatly due to changes in the environment, sufficient accuracy cannot be expected. Here, without using the signal strength of the tag, we use a method to locate by tag packet only. Since signal strength cannot be used, we reduce the signal effective distance of the tag and cover a small area with one tag. As a result, this area can be specified when a wireless packet of a tag is received. However, if there is overlap in the vicinity of the boundary of these areas, the wearable device sometimes receives signals from other areas evenly. Generally, upon receiving a tag packet, processing corresponding to the area indicating this tag is performed. Then, when the signal from the tag in another area is received occasionally, it immediately switches to the processing of the area, resulting in malfunction.

In order to accurately estimate the position, we developed an approach. For a beacon tag with the signal strength $s$, the device that has a distance $d$ from the tag, will receive the signal at a level as $X(s, d)$, and the packet error rate $P(X(s, d))$ is a normal distribution function defined as

$$
P\left(X(s, d)=\int_{a}^{X(s, d)} \frac{1}{\sqrt{2 \pi} \sigma(s)} e^{-\left\{\frac{(X(s, d)-\mu(X(s, d)))^{2}}{2 \sigma(s)^{2}}\right\}} d s\right.
$$

In an ideal environment, the average signal level $\mu(X(s))$ received by the device can be calculated as

$$
\mu(X(s, d))=\frac{K s}{d^{2}}
$$

where $K$ is a coefficient decided by sensitivity of the receiver. Based on the above formula, the PRR (packet received rate) $R(s, d)$ can be obtaioned by

$$
R(s, d)=1-X(s, d)
$$

Let us consider a typical case in Fig 7 As shown in Fig 7 there are two tags located in 40 and 80 position. In the section 40,80$]$, the packet received rate for both tags are not zero, so the device near tag 1 may receive the signal from tag 2 . It will casuse a wrong position identification sometimes. In order to avoid the problem, a PRR index $B_{i}$ is introduced to identify the spot area indicated by

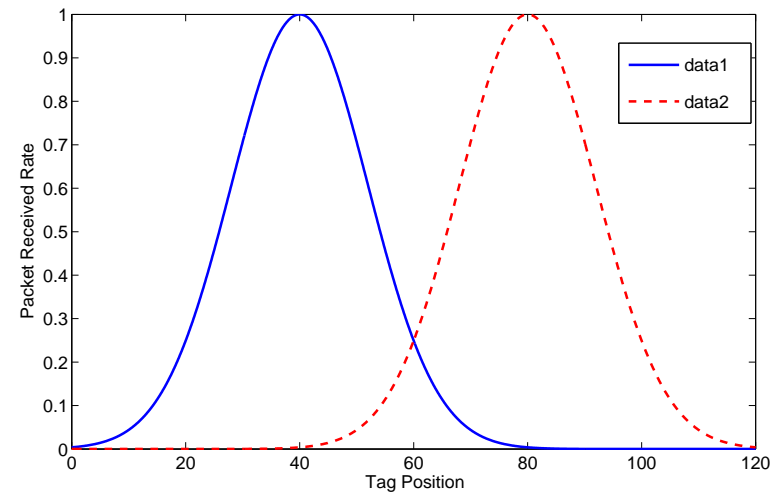

Fig. 7. The PRR of adjacent beacon tags

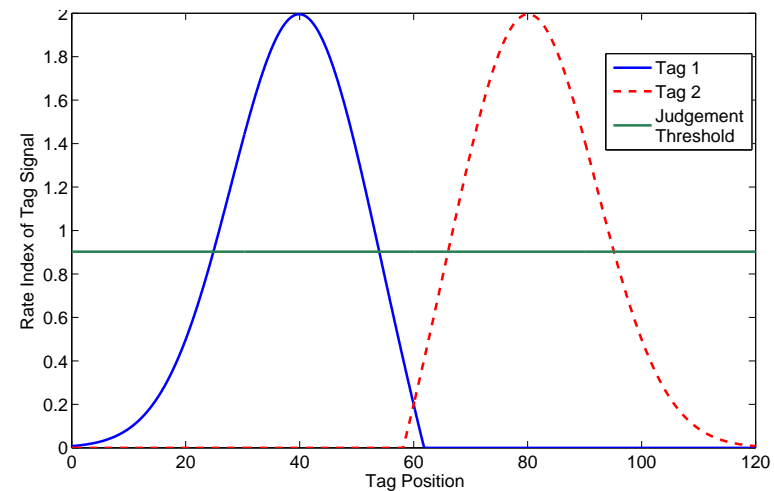

Fig. 8. Index PRR value of each spot area

tag $i$ as following

$$
B_{i}(x)=K_{1} * R\left(s_{i}, x-d_{i}\right)-K_{2} * \sum_{k=1 k \neq i}^{m} R\left(s_{k}, x-d_{k}\right)
$$

where $x$ is the identification spot, $s_{i}$ and $d_{i}$ are signal level and position for tag $i$, and $s_{k}$ and $d_{k}$ for tag $k . K_{1}$ and $K_{2}$ are weight factors. $K_{1}>K_{2}$. Fig 8 illustrates an example of PRR index. With a proper threshold $T_{i}$, the spot area can be distinguished from other spot area as shown in Fig 9

In a real environment, it is not easy to measure the PRR directly. As an implementation of the above approach, an algorithm for realizing the position identification is presented. In the system, each tag will send out packet signal at a fixed interval time $\tau$. The index $B_{I D_{i}}$ that represents the number or frequency of received tag signals $I D_{i}$ is defined. When a tag packet of $I D$ is received, for all $i, B_{I D_{i}}$ is modified by

$$
B_{I D_{i}}^{k+1}= \begin{cases}f\left(B_{I D_{i}}^{k}+\beta-\delta\left(t_{k+1}-t_{k}\right)\right) & I D_{i}=I D \\ f\left(B_{I D_{i}}^{k}-\beta-\delta\left(t_{k+1}-t_{k}\right)\right) & I D_{i} \neq I D\end{cases}
$$

where, $f(\cdot)$ is a function defined as

$$
f(x)= \begin{cases}\mu & (x>\mu) \\ x & (0 \leq x \leq \mu) \\ 0 & x<0\end{cases}
$$




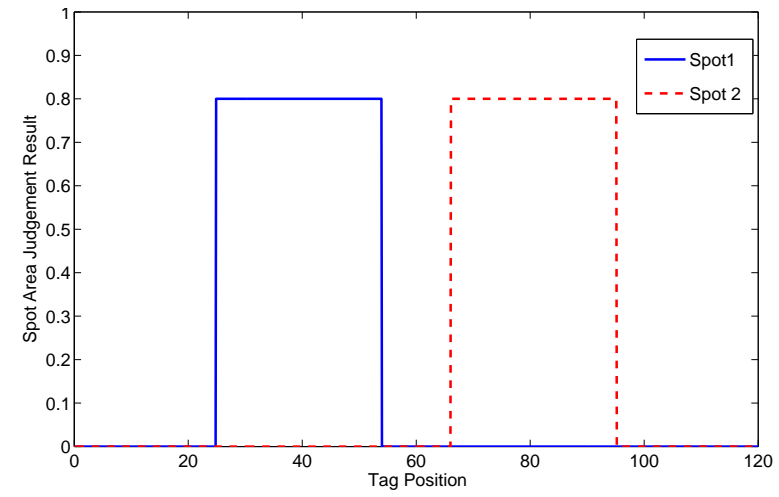

Fig. 9. The judgement result of each spot area

In the formula $[5, \delta$ reflects the elapsed effect of time. Fig 10 and Fig 11 illustrate the flow chart of the ID receiving frequency counting implementation. There are two threads for monitoring the tag receiving process. In the counting process thread, the tag signal frequency will be modified, when a new tag signal is received. The parameter $\mu$ is a saturation upper limit to avoid the too large value of the signal frequency.

In the position area identification process thread, there is a sort process that sorts the frequencies in ID list buffer and search for IDs with the maximum value and the minimum index value. For ID with minimum frequency value, we will remove the ID from the list buffer. A time reducing process is also carried out in the thread. When the maximum frequency value ID is more than a threshold omiga, it means that the tag signal is strong enough, and the ID message will be sent to the upper layer application. Fig 10 and Fig 11 show the above process in detail.

By actual measurement experiments, the identification accuracy of the tag area by this method has been improved from $72 \%$ to $90 \%$. Due to the introduction of this index, malfunction of the guide commentary was greatly lowered, and the provision accuracy of the guide commentary was greatly improved.

\subsection{Identification Approach of Orientation}

It may be a complex calculation for arbitrary situation device. In this guide service, we will give a condition for the situation of the device. This condition is used as a sign for starting explanation from the visitor. The constraint condition is that the visitor should hold the device straightly. For derivation of the calculation formulation, we will define two coordinates as shown in Fig 12 There are two coordinates defined, $O X Y Z$ and $O x y z$. $O X Y Z$ is fixed to ground and its $X$ axis points to the North Pole, and $-Z$ follows the gravity direction. Note the axis $X$ is not true north direction, there is a little difference between the North Pole and true north. The difference value can be compensated based on the latitude. Oxyz is fixed to the device as shown in Fig $12 \theta$ is the inclination angle of the device, and $\alpha$ is the orientation of the device. $a_{x}, a_{y}$ and $a_{z}$ are the acceleration sensor outputs on each axis. $M_{x}, M_{y}$ and $M_{z}$ are the terrestrial magnetism sensor outputs on each axis.

Because we have a constraint condition as a sign for starting guidance, the plane $X O Y$ and $x O y$ are at the same plane. This condition can be shown as

$$
a_{x}=0
$$

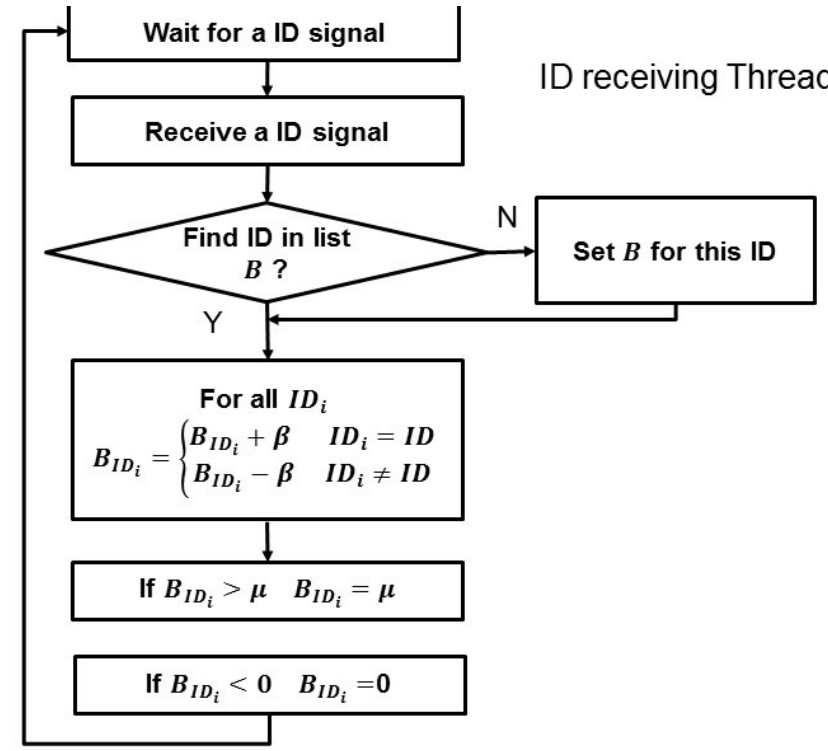

Fig. 10. The counting process thread

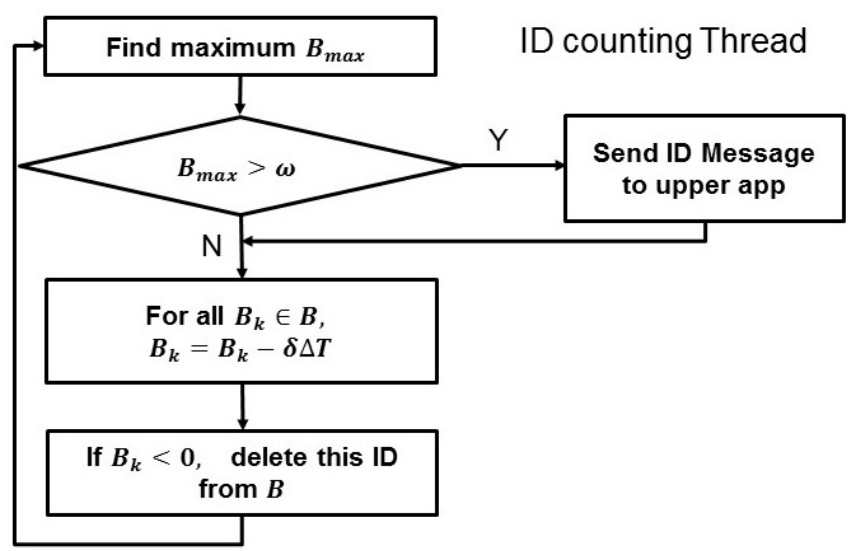

Fig. 11. The position area identification process thread

Because we use acceleration output to calculate $\theta$, the inclination of the device, the device should be in a still mode. To guarantee the condition, we will check if

$$
a_{x}^{2}+a_{y}^{2}+a_{z}^{2}-g^{2}=0
$$

is satisfied.

Under the constraint conditions, $\theta$ and $\alpha$ can be calculated from the outputs of acceleration sensor and terrestrial magnetism sensor. When the visitor holds the device without movement, the total acceleration is only gravity acceleration, therefore the $\theta$ can be obtained by

$$
\theta=\tan ^{-1}\left(\frac{a_{y}}{a_{z}}\right)
$$

When visitors require guidance service, they will hold the device with a proper tilt angle. Therefore, the tilt angle $\theta$ should be at a 
range

$$
0<\theta<\pi / 2
$$

Then we can calculate the orientation of the device as the followings

$$
\alpha=\tan ^{-1}\left(\frac{M_{y} \cos \theta-M_{z} \sin \theta}{M_{x}}\right)
$$

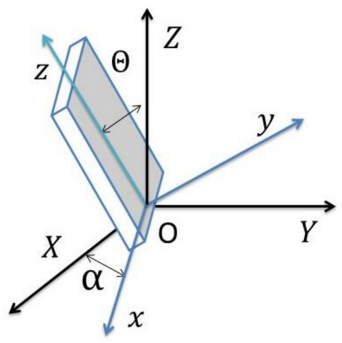

Fig. 12. The coordinates for ground and device

For the wearable guide device is equipped on the visitor's head, It is easy to obtain the orientation of visitor based on the orientation of the device. For the constraint condition mentioned above, the visitor will hold the device in front of him, therefore the orientation of the visitor $\beta$ is equal to the orientation of the device and can be shown as

$$
\beta=\alpha
$$

With the information from acceleration and terrestrial magnetism sensor, the algorithm for automatic guidance is given in Fig 13 When a visitor stands before an exhibit and holds the device up, the identification of the orientation will run. When the visitor holds the device straight, the $\mathrm{x}$ axis of the device should be paralleled with the ground. In this case, the $a_{x}$ should be zero. In real application, the output $a_{x}$ from acceleration sensor may not be exactly zero for electrical noise and little hand vibration. Therefore, we modify the condition as

$$
\left\|a_{x}\right\|<\epsilon_{1}
$$

where positive value $\epsilon_{1} \ll 1$. Another condition (7) will be modified as

$$
\left\|a_{x}^{2}+a_{y}^{2}+a_{z}^{2}-g^{2}\right\|<\epsilon_{2}
$$

where position value epsilon $n_{2} \ll 1$.

The value of $\epsilon_{1}$ and $\epsilon_{2}$ will be determined by some later experiments. Too small values for $\epsilon_{1}$ and $\epsilon_{2}$ may bring a better accuracy for identification, but may raise the time of calculation and make the visitors feeling tired. Therefore, it is important to adjust $\epsilon_{1}$ and $\epsilon_{2}$ for best performance.

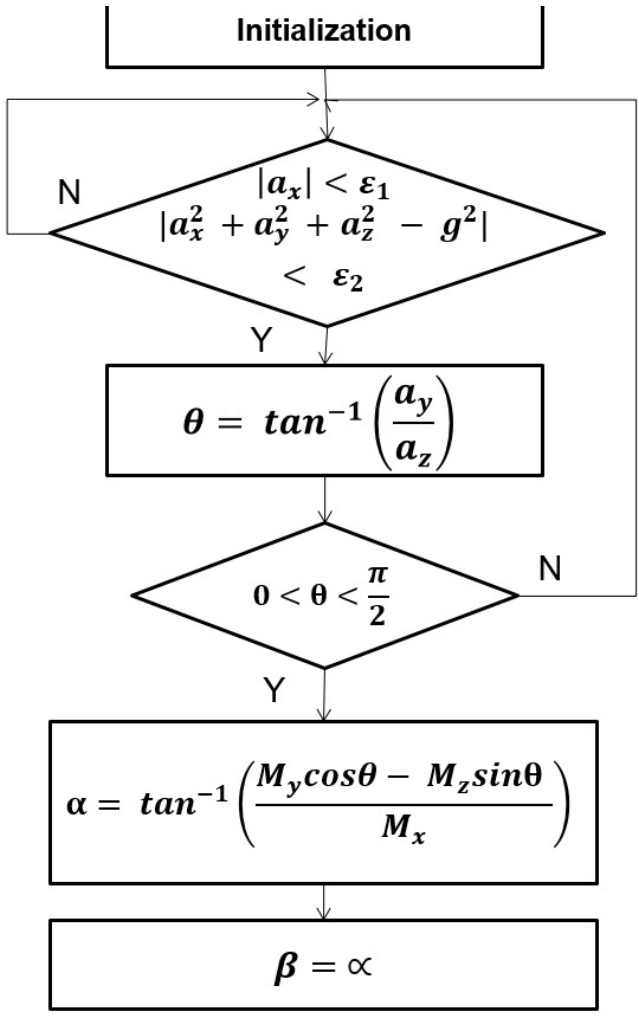

Fig. 13. The algorithm for identifying orientation

When the condition (12) and $(13)$ are satisfied both, we can start to calculate $\theta$, the inclination angle of the device by 8 . If the inclination angle $\theta$ satisfies the condition (9), it means that the device is in a good attitude for guidance service. Therefore, we can calculate the orientation of the device by $(10)$. Finally, we will obtain the orientation of the visitor by (11).

\subsection{Identification of the Tourist Status}

Fig 14 shows the general framework for identifying the status of the tourist. As illustrated in Fig 14, at first, the device will check if the device is within the area of an exhibition spot. If the device enter the area, then orientation calculation will be carried out. If there is an exhibit guidance for this orientation and position by checking the map DB of the exhibition are, the device will start to play the multimedia guidance content.

\section{CONCLUSIONS}

In this paper, a wearable guide device that can be worn on the ear and provide automatic guide service to tourists, has been presented. It is necessary to get the information about the position and orientation of the tourists for automatic guide service. In the device, there are a NFC beacon tag and a 6 axis acceleration and terrestrial magnetism MEMS sensor are used. To identify the guide spot location from the signal of the beacon tag, a calculation approach using the packet received rate is proposed, and an implementation of the approach is developed. To identify the orientation of the tourist, a set of formulas was derived based on the information from the MEMS sensor. A prototype of the wearable device has been 


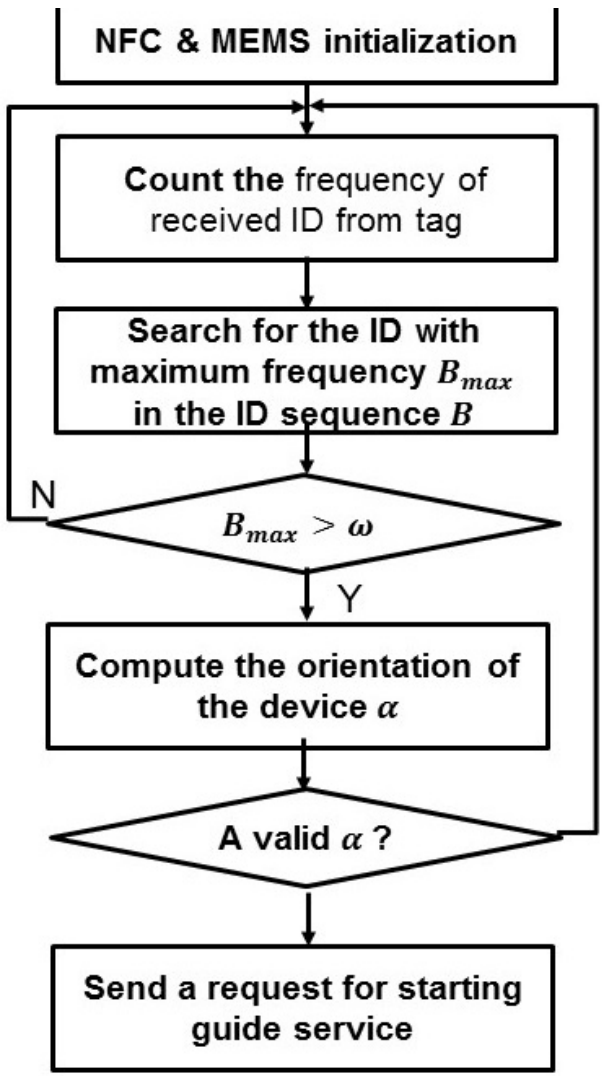

Fig. 14. The identification of the position and orientation

developed, and the performance for guide service was investigated via some experiments in a multi tag environment. The guide device and automatic service developed in this research are expected to provide a satisfactory performance for guide usages in near future.

\section{REFERENCES}

[1] Kusunoki, F., Sugimoto, M., Hashizume, H., Toward an interactive museum guide system with sensing and wireless network technologies, Wireless and Mobile Technologies in Education, 2002. Proceedings. IEEE International Workshop on Volume, Issue , Page(s): 99-102, 2002

[2] Li-Der Chou; Chia-Hsieh Wu; Shih-Pang Ho; ChenChow Lee; Jui-Ming Chen, Requirement analysis and implementation of palm-based multimedia museum guide systems , Advanced Information Networking and Applications, 2004. AINA 2004. 18th International Conference on Volume 1, Issue , 2004 Page(s): 352 - 357 Vol.1

[3] Bartneck, C., Masuoka, A., Takahashi, T., Fukaya, T., An Electronic Museum Guide in Real Use, Psychology of Aesthetics, Creativity, and the Arts, 1(2), 114-120, 2007

[4] Dawei Cai, Yuji Saito and Yoshihiko Abe, An Information Broadcasting System with Infrared data Communication Protocol, WSEAS Trans. on Communication, Vol.2(3), 228234, 2003
[5] Dawei Cai, Ryuuta Kawashima, Tadaaki Takehana and Haruki Takahashi, An Infrared Digital Contents Broadcasting Service for Mobiles, WSEAS Trans. on Communication, Vol.2(4), 73-78, 2005

[6] Dawei Cai, Realization of Autonomous Guidance Service by Integrating Information from NFC and MEMS, Proceedings of International Conference on Software Design Engineering 2014

[7] Dawei Cai, Developement of A New Wearable Device for Automatic Guidance Service , Proceedings of the ICCEPT 2015 